\title{
Reflets
}

Revue d'intervention sociale et communautaire

\section{Néo-libéralisme, inégalités et minorité : le point de vue d'un sociologue}

\section{Entrevue avec Donald Dennie}

\section{Rachid Bagaoui}

Volume 24, numéro 1, printemps 2018

URI : https://id.erudit.org/iderudit/1051519ar

DOI : https://doi.org/10.7202/1051519ar

Aller au sommaire du numéro

Éditeur(s)

Reflets, Revue d'intervention sociale et communautaire

ISSN

1203-4576 (imprimé)

1712-8498 (numérique)

Découvrir la revue

Citer ce document

Bagaoui, R. (2018). Néo-libéralisme, inégalités et minorité : le point de vue d'un sociologue : entrevue avec Donald Dennie. Reflets, 24(1), 25-39.

https://doi.org/10.7202/1051519ar d'utilisation que vous pouvez consulter en ligne.

https://apropos.erudit.org/fr/usagers/politique-dutilisation/ 


\section{Néo-libéralisme, inégalités et minorité : le point de vue d'un sociologue}

Entrevue avec Donald Dennie

\section{Présentation de la personne et de son organisme}

Donald Dennie est natif de la région de Sudbury. Il obtient un B.A. (histoire et philosophie) de l'Université Laurentienne en 1961. Après avoir été journaliste pour une dizaine d'années (L'Information à Sudbury et Le Droit à Ottawa et dans le Nord de l'Ontario) et avoir obtenu une M.A. (sociologie) de l'Université Carleton d'Ottawa en 1969, il intègre en 1974 les rangs du corps professoral de la Laurentienne (Département de sociologie), d'où il prendra sa retraite en juillet 2010. Il obtient une M.A. (histoire) de l'Université Laurentienne en 1986 et un Ph. D. (histoire sociale) de l'Université Carleton en 1989. Ses recherches principales portent sur la région de Sudbury. Deux livres, À l'ombre de l'INCO (Ottawa, Presses de l'Université d'Ottawa, 2001) et Une histoire sociale du Grand Sudbury: le bois, le roc et le rail (Sudbury, Prise de parole, 2017), constituent le fruit de ses recherches. Il continue également d'agir à titre de bénévole dans différents organismes de la région de Sudbury, dont le Salon du livre de Sudbury.

Cette entrevue, réalisée et rédigée par Rachid Bagaoui, porte sur le parcours de Donald Dennie, la découverte du marxisme, le néo-libéralisme et les inégalités ainsi que les inégalités en contexte minoritaire.

\section{La découverte du marxisme}

Reflets: J'aimerais retracer rapidement votre parcours professionnel et intellectuel. Vous étiez professeur de sociologie à l'Université Laurentienne, mais, avant ça, que faisiez-vous sur le plan professionnel? Qu'est-ce qui vous a amené à la sociologie?

Donald Dennie: J'étais journaliste pour le journal Le Droit, un quotidien d'Ottawa. J'ai fait, tout en travaillant, une maîtrise en sociologie 
à l'Université Carleton parce que je pensais que ça m'aiderait à comprendre davantage la société. Comme journaliste, je vais essayer de comprendre - en tout cas, pour moi - de comprendre davantage ce sur quoi j'écris des textes.

Alors voilà, j'ai fait ma maîtrise et ensuite je suis revenu dans la région de Sudbury pour faire de la recherche essentiellement et continuer comme journaliste en tant que correspondant $\mathrm{du}$ journal Le Droit. Et d'une chose à l'autre, le Département de sociologie m'a demandé de poser ma candidature à un poste - ce que j'ai fait - et j'y suis demeuré pendant 35 ans.

Reflets : Quel était votre champ d'intérêt quand vous avez commencé à faire la sociologie?

Donald Dennie : Assez curieusement, c'était la sociologie de la religion, et ça a vite changé lorsque j'ai commencé à faire la lecture de Karl Marx, surtout les Manuscrits de 1844, L'Idéologie allemande et ensuite Le Capital. J'ai alors commencé à m'intéresser aux classes sociales et aux distinctions à l'intérieur des communautés francophones, n'ayant jamais beaucoup communié - si je peux employer ce mot-là - à l'idée qu'il y ait une société franco-ontarienne et qu'il y ait une communauté, pour des raisons que je ne peux m’expliquer, je n'ai jamais pu communier à cette idéologie.

J'étais beaucoup plus intéressé à comprendre ce qui pour moi était la vraie, entre guillemets, "structure " de la société. Donc, j'ai fait beaucoup de travaux sur les communautés ou la population de langue française de la région de Sudbury principalement.

Reflets: On sait que Marx est un des théoriciens qui a abordé, à sa façon, la question des inégalités, de l'exploitation, de la domination. Comme vous appréciez les travaux de Marx, quel usage en avez-vous fait?

Donald Dennie : Je pense que c'était peut-être la mode à l'époque, début des années 1970, Marx était à la mode. Puis, quand j'ai fait ma maîtrise en sociologie, évidemment on m'a bourré le crâne avec des auteurs comme Parsons et ensuite un peu Durkheim et beaucoup Weber. 
Et Weber, je l'aimais beaucoup et je l'aime encore. J'aime encore Weber. Comme Marx est devenu à la mode dans les années 1970, j'ai commencé, par intérêt et par curiosité, à lire surtout ses écrits qu'on dit humanistes des années 1840, les Manuscrits de 1844. Et de là, j'ai lu Le Capital et j'ai beaucoup aimé sa façon d'analyser la société capitaliste ou, du moins, l'économie capitaliste. Ça m’a beaucoup interpellé.

Reflets: Comme étudiant et comme journaliste, quest-ce qui se passait autour de vous qui justifiait la référence ou le recours à Marx?

Donald Dennie: Au sein de la communauté dans laquelle je vivais, qui était francophone, il y avait certainement un renouveau culturel, et on remettait en cause beaucoup des assises culturelles et idéologiques de ce qu'on avait vécu, des assises qui étaient branchées sur l'église et toutes ces choses. On remettait tout ça en cause. Il y avait comme un remue-ménage intellectuel, pas seulement ici mais également au Québec et aussi dans les lectures que je faisais, dont celles de Gilles Bourque et d'autres qui essayaient de faire un mélange ou un amalgame de classes sociales et de nationalisme. Et voilà, j'ai donc commencé à faire ces lectures et regardé tout ce remue-ménage.

À l'époque, j'étais aussi un peu impliqué avec les animateurs socioculturels. Il y avait entre autres Jean-Robert Marcoux, qui était ici et qui essayait d'animer non seulement et pas uniquement ce qu'on appellerait les élites, qui ont toujours milité et travaillé au sein de la situation canadienne-française de l'Ontario, mais il voulait aussi animer les gens qu'il appelait "à la base ". Il a donc formé un comité de citoyens, et les gens s'intéressaient beaucoup plus au logement ou à l'alimentation, à des choses qui les touchaient de près, et non pas uniquement aux questions sur l'éducation, les droits linguistiques et tout ça. Et ça, ça m'a aussi beaucoup interpellé. Et tout ça mis ensemble, j'ai commencé à faire la lecture de Marx. 
Reflets: Mais une fois que vous êtes devenu professeur et, ensuite, quand vous avez pris votre retraite, est-ce que votre implication a cessé? Est-ce qu'il y a eu une coupure entre l'homme qui était impliqué dans la société " militante " et le côté académique?

Donald Dennie : J'ai continué à m'engager dans des organismes communautaires et j'ai continué à faire de la recherche et à publier dans la lignée dans laquelle je m’étais engagé au cours des années 1970. J’ai essayé de continuer mon engagement au niveau de la communauté et j'ai tenté de développer un volet qui m’avait toujours intéressé. Je vais appeler ça le volet artistique : dessiner, prendre des cours d'architecture, continuer des leçons de piano. C'est une partie de moi qui n'avait pas eu la chance d'être développée quand je travaillais à plein temps.

Reflets : Et est-ce que vous diriez que cette grille de lecture marxiste, vous l'avez gardée?

Donald Dennie : Oui, ça m’a toujours paru la grille d'analyse la plus pertinente, malgré tous les changements. Et je sais bien qu'au niveau académique, il y a des modes. Mais, moi, j'ai toujours pensé que l'analyse que Marx faisait des sociétés capitalistes était et demeurait fondée. Certes, la réalité est un peu plus complexe aujourd'hui au niveau des classes sociales. Entre autres, on ne peut plus parler essentiellement d'une classe ouvrière et d'une classe bourgeoise. Il y a toutes sortes de classes. Je sais que plusieurs auteurs ont tenté de faire une analyse en parlant de nouvelle petite bourgeoisie et tout ça. Mais, au fond, il me semble qu'il reste que la société est toujours semblable, même si elle se divise encore plus entre la grande bourgeoisie et une classe ouvrière qui est devenue beaucoup plus complexe, oui, beaucoup plus complexe.

Reflets : Vous avez publié dernièrement un livre intitulé "Une histoire sociale du Grand Sudbury: le bois, le roc et le rail ». Quelles sont les grandes conclusions que l'on peut dégager lorsqu'on se situe $d u$ point de vue marxiste des classes sociales appliquées aux travailleurs miniers de Sudbury? 
Donald Dennie : Bien, c'est évident qu'il y a eu un énorme changement quand, jusqu'au début des années 1980, les mineurs constituaient la classe ouvrière aristocratique, si l'on peut dire, qui faisait de bons salaires, avait certaines conditions de travail assez satisfaisantes, mais pas complètement. Il y a eu un énorme changement parce que les propriétaires des mines ont décidé d'automatiser. Donc, ils ont procédé à des congédiements, à des mises à la retraite de nombreuses personnes qui travaillaient dans les mines. On est passé d'environ 22000 mineurs à environ à 5000 aujourd'hui dans la région de Sudbury.

De plus, les travailleurs sont devenus davantage des cols blancs qui travaillent beaucoup plus dans les services, dans l'administration des services publics ou privés. Donc, il y a eu une restructuration de la classe sociale, mais ça n'empêche pas que la division ou la structure de la société, selon moi, demeure toujours la même, que de grands propriétaires - pour utiliser une belle expression de Marx — sont toujours propriétaires des moyens de production et, à cause de ça, peuvent et se doivent d'embaucher des gens qui vivent d'un salaire, alors que les grands bourgeois peuvent vivre d'une rente ou, si vous voulez, de certaines rentes.

Reflets: Certains disent qu'il n'y a pas que les classes sociales, il y a aussi des mouvements sociaux. Autrement dit, on peut revendiquer et demander la justice sociale, sans nécessairement être marxiste ou adopter une grille marxiste. Qu'en pensez-vous?

Donald Dennie : Je pense que la revendication pour les droits des travailleurs, ou du moins d'une certaine catégorie de travailleurs, n'est plus aussi à la mode, ou on ne la sent plus, on ne la voit plus autant qu'on la voyait dans les années 1970 et au début des années 1980. Mais, il reste que si l'on tient pour acquis que Marx a porté son analyse surtout sur la société capitaliste, il avait tout de même chez lui un fond plus humaniste. Je sentais que, dans les écrits de 1844 , il y avait chez lui un fond de pensée humaniste, où il essayait de mettre en valeur l'être humain. Et donc, pour moi, c'est ça; il y a 
des gens qui vont prôner la justice sociale parce que l'être humain a certains droits, certaines valeurs. Et donc, dans ce sens-là, je pense que la pensée de Marx est toujours déterminante. Moi, j'ai toujours un peu de difficulté avec les représentations parce que je vois ça comme des modes, et je pense que ce que je retiens de l'analyse de Marx, c'est qu'il faut toujours faire attention à ce qui apparaît, donc des représentations, et ce qui se retrouve en dessous de ce qui apparaît des représentations mentales.

\section{Les inégalités sous toutes ses formes}

Reflets : Comme marxiste, comment définiriez-vous les inégalités?

Donald Dennie : C'est un concept un peu abstrait qui a beaucoup de dimensions. Il y a certainement les inégalités au niveau des classes sociales qui se sont approfondies : quand on voit maintenant que les très grands propriétaires - les un pour cent qu'on appelle — ont une richesse extraordinaire et que les travailleurs s'appauvrissent ou ne réussissent pas à grimper dans la classe moyenne. En tout cas, il y a une disparité de plus en plus grande. Donc, il y a certainement une inégalité financière, économique et, puis, il y a des dimensions à l'intérieur de ça dont on pourrait parler.

Il y a aussi des inégalités au niveau du statut. Et là, je m’inspire peut-être un peu plus de Weber. Il y a des inégalités au niveau du genre, au niveau des groupes ethnoculturels ou - si je peux employer un ancien terme - au niveau des races, et les personnes de race noire ou jaune n'ont pas les mêmes statuts par rapport à celles de race blanche. Il y a des inégalités un peu partout. C'est donc un concept un peu vague, un peu abstrait. Et les auteurs vont analyser des dimensions selon leurs préférences ou leurs préoccupations.

Reflets: Comment expliquer les inégalités dans le contexte du capitalisme actuel (le néo-libéralisme)? Comment fonctionnent-elles? Quels liens peut-on faire entre les deux? 
Donald Dennie : Je pense que ce qu'on fait avec ce qu'on appelle le néo-libéralisme dans les sociétés occidentales surtout, c'est de procéder à la déréglementation. Par exemple, on a enlevé le pouvoir des syndicats et on a voulu laisser le marché de plus en plus libre. En effet, Thomas Piketty dans son livre intitulé Le capital au XXI siècle a démontré la forme économique des inégalités dans les sociétés capitalistes. Il me semble qu'à partir de ce moment-là, on a pu reprendre l'analyse critique de la société et commencé à recritiquer la société capitaliste qui, dans sa forme actuelle, va enrichir certaines personnes, certains groupes, surtout des Blancs, et va appauvrir le restant de la société.

Reflets : Quand on adopte une grille comme celle-là, marxiste évidemment, on a tendance à insister beaucoup sur les dimensions: capital, travail, promotion économique, etc., sachant aussi qu'il y a des inégalités culturelles, scolaires, etc. Que pouvez-vous nous dire là-dessus?

Donald Dennie : Bien, je pense que le concept d'inégalité a certainement été élargi à partir de l'inégalité économique et des classes sociales. Et, pour moi, le féminisme a beaucoup participé à faire connaitre davantage les inégalités entre l'homme et la femme dans les sociétés, et ça nous a permis de commencer à parler davantage de justice sociale. Il y a aussi tous les mouvements comme Black Lives Matter, celui des Autochtones au Canada tel Idle No More. Il y a un approfondissement de la notion d'inégalité à cause des mouvements sociaux, et les gens commencent à se prendre en main pour des raisons que je ne comprends pas toujours et que je ne pourrais pas analyser.

Reflets : Vous qui avez connu et écrit sur les deux périodes, soit celle d'avant les années 1970 et celle d'après, comment caractérisez-vous les visages de la pauvreté, des inégalités dans les deux contextes?

Donald Dennie : Je pense que la pauvreté s'est approfondie. Il me semble que de plus en plus les gens, que beaucoup de gens vivent de plus en plus dans la pauvreté parce que justement on est passé d'une période de manufacture, de grandes industries, à une autre forme de production, et ça a laissé de côté énormément de 
gens. Et maintenant, lorsqu'on met l'emphase sur l'austérité et qu'on veut couper les programmes sociaux - ce qu'on voit de plus en plus aux États-Unis et ce qu'on a vu au Canada jusqu'à un certain point - je pense qu'il y a énormément de gens qui tombent dans la pauvreté.

Ce que j'ai connu surtout, c'était le début du modèle de la société de bien-être, celle du bien-être social ou de la "welfare society ", comme on l'appelait dans les années 1940, et il y avait tous les programmes. Et ces programmes-là, du moins au Canada, la plupart existent encore, même si, dans plusieurs pays, on essaie de les réduire et même de les abandonner. Donc, il y a une forme de pauvreté qui existe encore, qui est à peu près la même qu'avant.

Je pense que lorsque j'étais plus jeune — je pense à la région ici - il y avait énormément de gens qui pouvaient subvenir à leurs besoins parce qu'ils étaient propriétaires de fermes et tout ça. Il y avait une possibilité au moins de subvenir à leurs besoins immédiats au niveau de l'alimentation et tout ça. Et ça, évidemment, ce n'est plus là.

Reflets: Selon certains, les inégalités demeurent; elles sont toujours là, mais sur une longue période, elles sont beaucoup plus en diminution qu'en augmentation à cause de ces garde-fous que sont les régulations sociales (assurance-emploi, assurance maladie, conventions collectives, etc.). Est-ce une lecture trop optimiste du capitalisme actuel?

Donald Dennie : Il est vrai qu'il y a plus de protection, certainement au Canada, dans les pays scandinaves, en Europe. Il y a beaucoup plus de protection en ce sens qu'on a une assurance santé et une assurance qu'on appelle " assurance-emploi " aujourd'hui, et il y a tout de même des programmes également. Mais je n'attribue pas ça au capitalisme. J'attribue ça à la démocratie, car on a tout de même un régime mi-démocratique — si je peux dire ainsi - une démocratie représentative et non pas de base. 
Ce n'est pas le capitalisme. C'est le fait qu'on avait une démocratie, que les gens ont demandé des droits, qu'ils ont obtenu des droits, qu'il y avait des syndicats qui ont lutté, qui ont combattu pour obtenir des droits, puis c'est à la suite de ces luttes-là, puis des mouvements sociaux qu'on a obtenu de la protection. Mais, pour moi, ce n'est pas le capitalisme parce que le capitalisme en soi, c'est un peu ce qu'on voit aujourd'hui. C'est une déréglementation où l'on veut que la classe propriétaire obtienne de plus en plus de droits, de bénéfices, de richesses, et les travailleurs...

Reflets : Lesystème démocratique dont vous parlez a-t-il rempli ses promesses en termes de combat contre les inégalités?

Donald Dennie : C'est un peu difficile à répondre. D'un côté, je crois que, dans la société actuelle, il y a une série de luttes afin que des gens puissent de plus en plus avoir de la protection basée sur une justice sociale et tout ça. De l'autre, il y a des gens qui veulent enlever ça parce qu'ils veulent - et là je parle des grands propriétaires et même des petits propriétaires - accaparer le plus possible les richesses ou les profits du travail. Mais, c'est toujours une lutte. J'aime beaucoup ce que Barack Obama dit régulièrement, c'est-à-dire que "The Arc of Justice " tend toujours vers une plus grande justice, une plus grande égalité. Pourtant, ce n'est pas quelque chose qui va juste de soi, et c'est toujours le résultat des luttes. Et là, aujourd'hui, ça dépend des sociétés, des nations, des Étatsnations dans lesquels on vit.

Je vois bien qu'aux États-Unis, on est en train de saboter presque tous les programmes sociaux, et qu'au Canada, il y a peut-être un équilibre un peu plus juste, mais l'équilibre est toujours en faveur des grands propriétaires ou des propriétaires parce que c'est ce qui fait rouler la machine. Et donc l'État, surtout quand un gouvernement libéral est au pouvoir, l'État et ces gens sont obligés d'essayer de trouver un équilibre quelconque qui va plaire à un côté et va plaire à l'autre. Mais, à long terme, l'État a toujours les intérêts fondamentaux de la classe bourgeoise en tête. 
Reflets : Le capital culturel est un déterminant important dans les inégalités. Quel est le rôle de l'éducation ( $p$. ex. de l'université) pour combattre les inégalités dans un contexte dominé par l'économie?

Donald Dennie : Je pense que les programmes qui sont plus rentables, " marchandables ", qui rapportent plus d'argent, sont beaucoup plus valorisés. Alors, oui, il y a des inégalités à l'intérieur des universités qui reproduisent les inégalités qu'on voit dans la société. Quant à l'éducation, je pense qu'on est rendu à un point où, comme on dit souvent, le B.A. équivaut aujourd'hui à un diplôme d'études secondaires à cause des demandes et des exigences du secteur économique. Mais je ne suis pas porté à dire que ça va réduire les inégalités. Ça va juste peut-être les renforcer. Ça va appuyer la valeur technologique, la valeur « marchandable » du travail, de la force de travail plutôt.

Reflets: J'aimerais aborder la question de la place des préjugés en général. Comme marxiste qui ne croit pas aux représentations, comment interprétez-vous ce phénomène des préjugés envers, par exemple, des immigrés, des minorités, des Autochtones, des femmes, des petites régions, etc.? Est-ce quil ne joue pas un rôle dans le renforcement des inégalités?

Donald Dennie : Oui, pour moi, ce n'est pas juste au niveau des représentations. Les représentations, je ne voulais pas dire tantôt que ça n'a pas de valeur. Je sais qu'il faut voir ce qu'il y a en dessous des forces matérielles, peut-être plus dans ce sens-là. Donc, les préjugés ont un impact au niveau individuel, au niveau psychologique individuel et collectif, et ça sert aussi à ça, comme on le voit quand - encore là je parle des États-Unis — les mouvements de droite essaient d'utiliser l'État afin de transformer les préjugés en programmes et en lois qui vont faire en sorte que certains groupes, ciblés par ces préjugés, soient pénalisés.

Et, certainement aux États-Unis, on le voit par les préjugés à l'égard des immigrants, des musulmans entre autres. Et juste hier, le gouvernement américain a annoncé qu'ils allaient déporter 200000 personnes du Vénézuéla qui avaient cherché refuge 
dans ce pays. Alors, ces préjugés-là, d'après moi, s'exercent à différents niveaux. Lorsqu'on s'en sert comme une force que je dirais matérielle ou politique, là ça devient encore plus fort, plus puissant. Et je ne veux pas dire que les préjugés au niveau individuel, par exemple le fait d'être une minorité, qu'on soit gai ou lesbienne, qu'on soit de religion musulmane, au niveau individuel, ça n'a pas de portée. Certainement, ça a une grande portée qui peut mener à toutes sortes de dépression, par exemple, ou de choses comme ça. Mais, quand ça entre dans la structure matérielle de la société au niveau des lois, des politiques et tout ça, c'est là que ça devient de plus en plus puissant selon moi.

\section{Reflets : Un mot sur les inégalités entre les hommes et les femmes?}

Donald Dennie : Je pense qu'il y a certainement une évolution grâce aux travaux des féministes. Maintenant, la question qui se pose est de savoir : est-ce que c'est une évolution qui a privilégié davantage les Blancs ou les Blanches que les Noirs ou les Noires? Par exemple, on voit par le mouvement \#MeToo aux États-Unis que les femmes commencent à dénoncer des gens, des hommes qui les ont agressées. Mais, pour moi, quand je regarde ça, c'est surtout un mouvement qui est le fait des femmes qui ont tout de même certains... Par exemple, ce sont des comédiennes, des actrices, des personnes qui peuvent peut-être se permettre de dénoncer. Alors que les femmes qui nettoient des chambres d'hôtel, qui n'ont pas de permanence, qui n'ont pas de sécurité de travail et qui se font probablement agresser, ces personnes-là, on n'en entend pas parler beaucoup.

Mais, cela étant dit, oui, il y a certainement selon moi une évolution. Je le vois chez les petites filles, par exemple, et on le voit aussi depuis les années 1980. Ce que j'ai vu à l'université, c'est l'arrivée assez massive de femmes, de jeunes filles comme étudiantes, ce qu'on ne voyait pas auparavant. Et je pense même qu'aujourd'hui les filles, les femmes représentent le plus grand pourcentage au niveau des étudiantes et étudiants universitaires et peut-être même collégiaux. Donc, oui, il y a certainement une 
évolution. Mais, encore là, je pense qu'il faut faire attention à la question de la couleur de peau et tout ça...

Reflets : Si vous aviez à résumer votre pensée à une lectrice ou à un lecteur qui voudrait comprendre les causes des inégalités, qu'est-ce que vous diriez en général?

Donald Dennie : Bien, pour revenir à ce que je disais au début, les causes sont d'abord multiples. Et ce qui est encourageant, c'est de voir que dans le contexte actuel, du moins dans les sociétés démocratiques, il y a tout de même des mouvements importants afin de réduire les inégalités presque à tous les niveaux. Ça semble être plus difficile au niveau économique, mais certainement à d'autres niveaux; les inégalités au niveau du genre, les inégalités entre les personnes gaies et lesbiennes, LGBT, etc., il y a certainement à ce niveau-là un mouvement ou des mouvements pour réduire les inégalités. Il y en a eu un au Canada pour réduire les inégalités entre les Autochtones et le reste de la société. Donc, les inégalités et les causes sont multiples, mais il y a tout de même - et ce serait difficile de ne pas les voir - il y a beaucoup de mouvements, il y a beaucoup de gens qui se préoccupent et qui luttent pour réduire les inégalités à presque tous les niveaux.

\section{Les inégalités en contexte minoritaire}

Reflets : J'aimerais terminer cette entrevue en abordant la question de la minorité linguistique. Vous avez écrit un article qui était intéressant, où vous remettiez en cause l'idée de la communauté. On parle beaucoup de communauté, comme si elle allait de soi, comme sil s'agissait d'un bloc homogène, etc. Mais on sait qu'à l'intérieur, il y a des classes, des rapports hommes-femmes, des inégalités régionales, etc. J'aimerais savoir pourquoi remettre en cause cette idée de communauté?

Donald Dennie : Je pense que, parce que j'étais journaliste, j’ai vu les différences à l'intérieur de ce qu'on appelle la société ou la communauté franco-ontarienne qui, pour moi, est une forme d'idéologie qui a des fonctions politiques, qui a des fonctions idéologiques 
évidemment. Mais, je voyais bien et j'ai toujours vu qu'à l'intérieur, il y avait des différences. Et, pour moi, ça m'a toujours surpris qu'on ne voie pas ça, parce que, moi, c'est ce que j'ai vu, c'est ce que j'ai observé et c'est ce que j'ai vécu. Et donc, un peu à partir de mon vécu, et de ma lecture de Marx, je voyais bien que les gens qui transmettaient l'idéologie parvenaient d'une certaine strate de la société et que ce n'était pas tout le monde qui adhérait au discours.

Récemment, on m'a demandé, dans le cadre du livre que j’ai écrit et publié, L'histoire sociale du Grand Sudbury, pourquoi je n'ai pas mis l'emphase sur la transition entre le Canada français et le franco-ontarien. Ma réponse, c'est que cette transition est importante pour certains milieux : les milieux universitaires, le milieu journalistique, les milieux culturels. Mais si vous allez demander à un travailleur comment il a vécu cette transition, il ne saura pas de quoi je parle.

Donc, pour moi et dans ce sens-là, c'était peut-être que - et je ne sais trop pourquoi — j'ai vécu la même chose que bien du monde, mais j'ai toujours éprouvé de la difficulté à adhérer à ça parce que je voyais, je vivais d'autres choses. Je dis probablement grâce au fait que j'ai été journaliste et que j'ai pu voir beaucoup d'autres réalités. Entre autres, j'ai commencé en tant que journaliste, comme on dit le beat. Je couvrais la police et les tribunaux, et ça m'a fait voir le sous-sol, si tu veux, de la société. Ensuite, j'ai couvert la politique qui m’a fait voir le théâtre, parce que la politique, il y a beaucoup de théâtre làdedans.

Reflets : Vous pensez à Goffman quand vous parlez de la politique comme théâtre?

Donald Dennie : J'ai beaucoup aimé Goffman, et la lecture de ses travaux m'a fait voir la représentation de soi dans la vie quotidienne et le fait qu'il y a des masques. Eh oui, la société est un peu un théâtre et, pour moi, quand j'ai couvert la politique pendant un an, c'est devenu tellement évident parce que les gens peuvent dire 
des choses publiquement et, quand on leur parle en privé, par exemple autour d'une bière, ils vont dire le contraire. Mais, j'ai pu voir à travers mes voyages en Ontario les différentes réalités qui ne sont pas et qui n'ont rien à faire avec une communauté, une société franco-ontarienne.

Reflets : Comment caractériser les inégalités en contexte minoritaire? Est-ce qu'il y a quelque chose de différent par rapport à ce qu'on vit dans l'ensemble?

Donald Dennie: Encore, j’ai beaucoup de difficultés avec le terme minorité ou majorité. Donc, on est une minorité linguistique, mais est-ce qu'on est une minorité économique? J'en doute. Les francophones, les Franco-Ontariens, qu'on appelle la minorité, ont tout de même accès aux écoles. Ils ont presque toujours eu accès à l'école. Il y a sans doute des conséquences et des répercussions du fait qu'on parlait français, certainement par rapport aux anglophones. Mais, je vois que ces différences s'amenuisent de plus en plus. Quant aux anglophones, cette majorité n'est plus uniquement anglophone, parce que bien qu'elle le soit jusqu'à un certain point, dans le sens où la plupart des gens parlent l'anglais, ce n'est plus la majorité britannique d'autrefois parce qu'il y a peut-être le tiers maintenant de la population qui est de souche immigrante, donc qui apprend à parler l'anglais parce qu'à travers le monde on parle anglais. Mais voilà, de dire majorité et minorité, pour moi, c'est encore trop abstrait. J'ai de la difficulté avec ces concepts stricts. Oui, les parlants français sont désavantagés au niveau de la langue, s'ils veulent avoir des services en français, s'ils veulent avoir des affiches en français... Oui, il y a cette masse défavorisée à ces niveaux. Est-ce que ça se traduit au niveau économique? Là, je n'en suis pas certain. C'était peut-être vrai il y a 50 ans, mais je pense que ça l'est beaucoup moins aujourd'hui. Les gens ont accès à des diplômes. 
Reflets: Quels sont, selon vous, les déterminants des inégalités au sein de cette communauté?

Donald Dennie : Pour moi, c'est à peu près les mêmes déterminants que dans la société en général. Je ne vois pas de déterminants spécifiques. Il y en a qui sont inégaux pour des raisons économiques. Il y en a qui sont inégaux parce qu'ils sont, encore là, parce qu'ils appartiennent à une communauté gaie ou lesbienne. On a des préjugés à leur égard. Les femmes, oui, on sait qu'elles font moins de salaire en général, mais j'ai de la difficulté à voir qu'il y a des déterminants spécifiques au niveau des francophones qui ne sont pas des déterminants que l'on voit dans l'ensemble de la société.

Reflets : Merci pour cette entrevue très enrichissante.

Donald Dennie : Ça m’a fait plaisir. 